\title{
Depresión, ansiedad, apoyo interpersonal y dolor autopercibido en pacientes con síndrome de fibromialgia*
}

\section{Depression, Anxiety, Interpersonal Support, and Self-Perceived Pain in Fibromyalgia Syndrome Patients}

\author{
Juan Esteban Lozano** \\ ORCID: 0000-0001-5688-5061 \\ Tatiana Leal \\ ORCID: 0000-0002-9411-3983
}

Universidad Surcolombiana. Colombia

Marvin Peña-Altamar ORCID: 0000-0002-9187-1877

Clínica Privada Especialista en Reumatología de la ciudad de Neiva, Colombia

Silvia Olivera ORCID: 0000-0003-4649-9700

Nicolás Núñez

ORCID: 0000-0001-7424-3329

Universidad Surcolombiana. Colombia

Recibido: 3 de julio de 2019 Revisado: 25 de octubre de 2019 Aceptado: 30 de marzo de 2020

\section{Resumen}

El síndrome de fibromialgia (SF) es una patología que genera gran dificultad en los movimientos, fatiga y dolor en partes del cuerpo, afectando severamente la calidad de vida. El objetivo de este estudio fue determinar los indicadores de depresión, ansiedad, apoyo interpersonal, dolor autopercibido y su relación, en una muestra de pacientes con SF en Colombia. A 100 pacientes se les aplicaron las siguientes pruebas: Inventario de Ansiedad Rasgo Estado, Escala de depresión de Zung, Cuestionario para evaluación del apoyo interpersonal, y la Escala analógica del dolor. Se reportaron niveles moderados de depresión y ansiedad, dificultades específicas en el apoyo social y niveles altos en dolor autopercibido. Las puntuaciones en la escala de depresión se asociaron positivamente con la ansiedad estado y rasgo, pero se asociaron negativamente con el apoyo social. En conclusión, la salud mental, el apoyo interpersonal y el dolor autopercibido se encuentran afectados en los pacientes. Se requiere ajustar los protocolos de atención clínica con el propósito de conservar y potenciar la salud integral en los afectados.

Palabras clave: Síndrome de Fibromialgia, Depresión, Ansiedad, Apoyo Interpersonal, Dolor.

Artículo de investigación. Citar como: Lozano, J. E., Leal, T., Peña-Altamar, M., Olivera, S. y Núñez, N. (2020). Depresión, ansiedad, apoyo interpersonal y dolor autopercibido en pacientes con síndrome de fibromialgia. Diversitas: Perspectivas en Psicología, 16(2), 399-411. https:// doi.org/10.15332/22563067.5007

Autor de correspondencia: Juan Esteban Lozano Plazas, Psicólogo, Magíster en Neuropsicología Clínica, Investigador - Grupo Carlos Finlay de la Facultad de Ciencias de la Salud, Universidad Surcolombiana. Correo electrónico: juan.lozano@usco.edu.co. Dirección postal: Facultad de Ciencias de la Salud, Universidad Surcolombiana, Dirección: Calle 9 carrera 14, Neiva (Huila), Colombia. 


\section{Abstract}

The fibromyalgia syndrome (FS) is a pathology that generates great difficulty in movements, fatigue, and pain in different parts of the body, affecting severely the quality of life. This study was aimed to determine the indicators of depression, anxiety, interpersonal support, self-perceived pain, and their relationship in a sample of patients with Fs in Colombia. The following tests were applied to 100 patients: State Trait Anxiety Inventory, Zung's Depression Scale, Interpersonal Support Evaluation List, and Analogue Pain Scale. Moderate levels of depression and anxiety were reported, specific difficulties in social support, and high levels of self-perceived pain. Depression scores were positively associated with state and trait anxiety scores but negatively associated with social support. In conclusion, mental health, interpersonal support, and self-perceived pain are affected in Fs patients. It is necessary to adjust the clinical care protocols to preserve and enhance the integral health of those affected.

Keywords: fibromyalgia syndrome, depression, anxiety, interpersonal support, pain.

\section{Introducción}

La Organización Mundial de la Salud en el año 1992 reconoció la fibromialgia como una nueva entidad clínica (Haworth Editorial Submission, 1993), denominada Síndrome de Fibromialgia (SF). Este síndrome se caracteriza por presentar altos niveles de dolor y de manifestación crónica, sin una etiología claramente identificada (López y Mingote, 2008), puede presentar síntomas como rigidez por las mañanas, hormigueo o adormecimiento en las manos y los pies, dolores de cabeza, abdominalgia, alteraciones cognitivas y de memoria (Souza et al., 2018), síndrome de intestino irritable, ansiedad y depresión (Casado, 2015).

La fibromialgia afecta en promedio al $2.10 \%$ de la población mundial; al 2.31 \% de la población europea; y al $2.40 \%$ en la población española. Asociado a esto, se encuentra que los costos económicos son altos y en España se han estimado en más de 12.993 millones de euros anuales (Cabo-Meseguer, Cerdá-Olmedo y TilloMatta, 2017). En Colombia no se cuenta con estadísticas epidemiológicas de este síndrome (Cardona-Arias, León-Mira y Cardona-Tapias, 2013).

El SF aún carece de un consenso científico en cuanto a sus mecanismos de aparición y curso de la enfermedad. Evidencias sugieren que factores genéticos y ambientales predisponen a las personas a desarrollar depresión o fibromialgia (Gracely, Ceko y Bushnell, 2012), y no porque los genes sean directamente responsables, sino porque incrementan el riesgo de padecimiento en respuesta a eventos circunstanciales (Maletic y Raison, 2009). Algunos autores dan importancia a la existencia de una falla disfuncional en el sistema nociceptivo, encargado de la detección de amenazas a través de los mecanismos de alerta, dolor y estrés; generándose diferentes respuestas a estímulos externos. Estas respuestas se caracterizan por un bajo umbral en la sensibilidad del dolor y una estimulación rápida en respuestas de amenaza ante el ambiente (Lautenbacher, Rollman y McCain, 1994). No obstante, se han identificado en estudios neurobiológicos, datos que sugieren que la fibromialgia y la depresión comparten mecanismos biológicos, estos son: alelos de genes poco funcionales que regulan la señalización de las citoquinas sobre las vías monoaminérgicas, glutamatérgicas, señales neurotróficas, mecanismos opioides y respuestas inflamatorias (Maletic y Raison, 2009). Además, ciertos factores se asocian con su aparición, por ejemplo, la presencia de $\mathrm{SF}$ en familiares de primer grado (Arnold et al., 2004; Kato, Sullivan, Evengård y Pedersen, 2006), presencia de enfermedades con cuadros de dolor significativo y con mayor prevalencia en el género femenino (Casado, 2015). 
Actualmente la depresión y la ansiedad son patologías que han aumentado en la comunidad en general, todo esto debido a condiciones sociales más exigentes a nivel laboral y económico, aunque en el SF estas condiciones tienen mayor frecuencia y repercusión (Revuelta-Evrard, Segura-Escobar y Paulino-Tevar, 2010). Las limitaciones, que son generadas por el dolor constante y la fatiga, hacen que las personas con síndrome de fibromialgia se sientan poco productivas y no puedan desempeñar sus labores con normalidad. En el área laboral, los pacientes perciben escaso apoyo social y puede deberse al desconocimiento y poca sensibilización sobre el tema, lo que lleva a que estas personas tengan escasas relaciones personales en su trabajo, dada la incomprensión sobre la menor efectividad con que pueden realizar sus labores (BrionesVozmediano, Ronda-Pérez y Vives-Cases, 2015).

Para estas personas, mantenerse en el área laboral les ayuda a evitar el aislamiento social y reduce el impacto de la enfermedad sobre la calidad de vida (Vicente-Herrero, Terradillos-García, CapdevilaGarcía, Ramírez-Íniguez de la Torre y López-González, 2011). Esto, debido a que la actividad laboral los distrae de temas como la autopercepción de los síntomas, el grado de disfuncionalidad o la pérdida total o parcial de actividades de la vida cotidiana.

En Colombia, se han realizado pocos estudios relacionados con el SF asociado a la calidad de vida, el funcionamiento psicológico y el estado de salud. Específicamente, en investigaciones realizadas en la ciudad de Medellín, se encontró que los pacientes con SF que tenían poca escolaridad, edad mayor, sedentarios y sin participación social, presentaban mayor deterioro físico (Cardona-Arias, León-Mira y Cardona-Tapias, 2014). Así mismo, varios estudios han evidenciado que la calidad de vida en el SF se ve afectada por la falta de energía, el dolor, las alteraciones de sueño, la depresión y la ansiedad (Amaro, Martín, Anton y Granados, 2006; CardonaArias, León-Mira y Cardona-Tapias, 2013).

Finalmente, es importante tener en cuenta que aún en la actualidad no hay tratamiento para curar o contrarrestar el progreso del SF. Conociendo los síntomas y su severidad, el equipo médico entabla un proceso terapéutico, que puede ir modificándose acorde al tiempo y malestar autopercibido por la persona diagnosticada. Esto en apoyo con un equipo interdisciplinar que aborde estrategias terapéuticas para la mejora de patologías que emergen en personas diagnosticadas con fibromialgia, como la ansiedad, el estrés y la depresión, en función de generar bienestar y mejora en la calidad de vida de estos pacientes.

Con lo anterior, se formula la siguiente pregunta de investigación: ¿Cuáles son los indicadores de depresión, ansiedad, apoyo interpersonal, dolor autopercibido y su relación, en una muestra de pacientes con Síndrome de Fibromialgia en Colombia?

\section{Método}

\section{Diseño}

La presente investigación se enmarcó dentro un enfoque cuantitativo con un diseño no experimental, con un fin descriptivo, correlacional y de tipo transversal. El estudio se realizó sin manipular deliberadamente las variables y se describen relaciones entre variables en un momento determinado.

\section{Participantes}

La muestra estuvo conformada por 100 personas, 96 mujeres y 4 hombres, diagnosticados con síndrome de fibromialgia (SF) que asistieron de forma ambulatoria a la consulta externa de medicina interna y reumatología de una Institución Promotora de Salud (IPS) en la ciudad de Neiva (Colombia). El promedio de edad fue de 53.7 años ( $D E=12.4$ ), y escolaridad de 8.8 años $(D E=4.5)$. Los criterios de inclusión que se tuvieron en cuenta para realizar el ingreso al estudio fueron los siguientes: 1) presentar diagnóstico de fibromialgia, hecho por un reumatólogo o médico internista con experiencia acreditada en $\mathrm{SF}$ utilizando los criterios ACR 2010 para SF, 2) que asistieran de forma ambulatoria a la consulta externa de medicina interna y reumatología, 3) tener entre 18 y 85 años de edad, 4) saber leer, escribir y 5) que aceptara participar en el estudio firmando el consentimiento informado. Entre los criterios de exclusión se tuvo en cuenta: 1 ) no poseer enfermedades terminales con pronóstico igual o inferior a seis meses de vida, 2) antecedentes neurológicos 0 
psiquiátricos ántes del diagnóstico de $\mathrm{SF}, 3$ ) encontrarse hospitalizado al momento de la evaluación, 4) historial de discapacidad intelectual y 5) ser menor de 18 o mayor de 85 años.

\section{Instrumentos}

\section{Entrevista inicial}

En la entrevista inicial se recogió la información relacionada con los datos sociodemográficos y clínicos del paciente (edad, escolaridad, género, ocupación, estrato socioeconómico, estado civil, entre otros), años que padece y que fue diagnósticada la enfermedad. Además de esto, se hizo un registro clínico realizado por el médico especialista donde se registró los medicamentos que recibía actualmente para su enfermedad, la comorbilidad asociada y el diligenciamiento de la Escala Visual Analógica del dolor VAS para el SF.

\section{Cuestionario de salud del paciente (PHQ9)}

Es un examen auto administrado empleado para detectar síntomas de trastornos mentales. El PHQ-9 es uno de los módulos del PHQ y evalúa específicamente depresión (Wulsin, Somoza y Heck, 2002). La prueba consiste en nueve ítems, en donde, se le pide al paciente que marque una de las opciones de respuesta que oscilan entre cero y tres, dependiendo de la frecuencia con que la persona ha experimentado los síntomas descritos en el ítem en las dos semanas anteriores al examen. Para calificar la prueba, las respuestas son totalizadas, con un rango entre 0 y 27 (Silber y Tippett, 1965). Hay una pregunta adicional al final de la prueba donde se le pide al participante, si ha respondido que tenía problemas en las preguntas precedentes, que señale el nivel de dificultad en cualquiera de los problemas señalados y que han afectado su habilidad para hacer su trabajo o para cuidar de su casa o de otros. Las respuestas van desde "Ninguna dificultad" a "Dificultad extrema", y esta última pregunta no se incluye para calcular el puntaje total de la prueba. El PHQ-9 se usa en unidades de asistencia primaria y puede administrarse en español o en inglés (Silber y Tippett, 1965).

\section{Inventario de Ansiedad Rasgo-Estado (IDARE)}

Esta conformada por dos escalas de autoevaluación separadas que se utilizan para medir dos dimensiones distintas de la ansiedad: 1) la denominada Ansiedad-Rasgo (propensión ansiosa relativamente estable), que consta de 20 afirmaciones en las que se pide a los individuos describir cómo se sienten generalmente, y 2) la Ansiedad-Estado (condición emocional transitoria), que consiste en 20 afirmaciones, se requiere al sujeto que indique cómo se siente en un momento dado (Spielberger y Guerrero-Diaz, 1975). Los reactivos en cada escala son de tipo Likert de cuatro opciones que van desde "no en lo absoluto" a "mucho". Se ha reportado una alta confiabilidad (alfa de Cronbach de 0.83 para el IDARE-R y de 0.92 para el IDARE-E). El IDARE es aplicable en adultos, estudiantes y pacientes. La dispersión de posibles puntuaciones para el Inventario del IDARE varía desde una puntuación mínima de 20 , hasta una máxima de 80 (Pérez, 2005).

\section{Escala para evaluar el apoyo interpersonal (ISEL-12)}

Consistió en una lista de 12 afirmaciones relacionadas con la disponibilidad percibida y potencial de los recursos sociales. Los ítems que comprenden el EEAl se agrupan en tres subescalas de cuatro ítems. La subescala "Apoyo Social Instrumental" mide la disponibilidad percibida de ayuda material; la subescala de "Apoyo Social Percibido" evalúa la disponibilidad percibida de otra persona con la cual se puede hablar de los problemas propios; y la subescala de "Redes sociales" mide la disponibilidad percibida de las personas con las cuales uno puede realizar actividades (Moreno, Arango-Lasprilla y Rogers, 2010). El ISEL-12 se ha empleado ampliamente en la investigación en salud.

\section{Escala de depresión de Zung}

Fue diseñada para evaluar el nivel de depresión en pacientes diagnosticados con algún desorden depresivo. Es una encuesta corta que se auto administra para valorar que tan deprimido se encuentra un paciente. Hay 20 asuntos en la escala que indican las 4 características más comunes de la depresión: 
el efecto dominante, los equivalentes fisiológicos, otras perturbaciones y las actividades psicomotoras (Campo, Diaz y Rueda, 2006).

\section{Escala visual analógica del dolor (VAS)}

Elaborada por Scott Huskinson en 1976, facilita la medición de la intensidad del dolor que describe el paciente. Se trata de una línea horizontal o vertical de 10 centímetros. Representa en continuo la experiencia de dolor, en cuyos extremos está descrita, "sin dolor" en un extremo y "el peor dolor imaginable" en el otro, no contiene otra descripción a lo largo de la línea. Al paciente se le pide indicar sobre el continuo de la línea la intensidad de su sensación de dolor. La escala reporta buena confiabilidad y validez (De Loach, Higgins, Caplan y Stiff, 1998). Un puntaje por debajo de 4 significa dolor leve o leve-moderado, un puntaje entre 4 y 6 indica dolor moderado-grave, y un puntaje superior a 6 indica la presencia de dolor muy intenso (Ahlers, Van der Veen, Van Dijk, Tibboel y Knibbe, 2010).

\section{Procedimiento}

El comité de ética de la Universidad Surcolombiana proporcionó el aval para realizar el estudio. Los pacientes con SF asistían a la consulta externa de reumatología, quienes cumplían con los criterios del estudio, se les explicaba el objetivo de la investigación y una vez mostraban interés, se programó una cita para que asistiera a las instalaciones de la IPS, todos los participantes dieron su consentimiento informado. El estudio consistió en una entrevista y medidas de autoinforme administradas por un psicólogo. La aplicación del protocolo de evaluación tardó aproximadamente 40 minutos en completarse.

\section{Análisis estadísticos}

Se creó una base de datos codificada en el paquete estadístico SPSS para Windows versión 20 (2011). Con el fin de determinar los niveles de prevalencia de depresión, ansiedad y dolor autopercibido, como para el apoyo interpersonal, se calcularon las medias $(M)$ y desviaciones estándar $(D E)$ para cada una de estas pruebas. Además se establecen las respectivas interpretaciones de los promedios obtenidos por la muestra. Con el objetivo de determinar si existió una relación entre las variables a estudio (depresión, ansiedad, dolor autopercibido, apoyo interpersonal), se realizaron una serie de análisis de correlaciones de Pearson con el fin de conocer la fuerza que influyen unas sobre otras. En todos los análisis estadísticos se tomó el valor $p<0.05$ como referencia para indicar el nivel de significancia.

\section{Consideraciones éticas}

No existió ningún riesgo potencial para los participantes del estudio. La presente investigación incluyó pruebas psicológicas de papel y lápiz que se utilizan de manera rutinaria en la práctica clíni$\mathrm{ca}$, donde no se incluyen situaciones embarazosas ni de riesgo físico para los participantes. El riesgo psicológico fue mínimo, aunque fue posible que las pruebas produjeran fatiga o frustración en algunos de los participantes. Para reducir este riesgo, el evaluador identificó los signos de fatiga, ansiedad o frustración y realizó descansos con el fin de esperar la recuperación del paciente. Para proteger la confidencialidad de los datos, se asignó números para identificar a los participantes del estudio, y las pruebas fueron guardadas en un archivador con llave en la oficina del investigador principal del estudio. A todos los participantes del presente estudio se les solicitó que firmarán un consentimiento informado en el cual se describía el propósito del estudio y los procedimientos a realizar, se informó que la participación en el estudio era absolutamente voluntaria y sin ningún costo para la persona, se dejó claro que la persona podría negarse a participar en el estudio o retirarse en cualquier momento sin tener que dar explicaciones, se estableció que la investigación no representaba ningún riesgo físico para la persona, de igual forma que la persona seleccionada no recibiría ningún beneficio económico por participar en el estudio, y desde luego que la información personal sería manejada de forma confidencial y únicamente los investigadores del presente estudio erán las personas que podían tener acceso a la misma. Por último, el estudio contó con el aval del comité de bioética de la Facultad de Salud de la Universidad Surcolombiana. 


\section{Resultados}

\section{Características sociodemográficas y clínicas}

La tabla 1 muestra las principales características sociodemográficas recolectadas en la entrevista inicial.

La muestra estuvo conformada en su mayoría por mujeres, quienes se ocupaban de las labores del hogar ( $55 \%$ ), que constituían familias nucleares (39\%) o monoparentales (32\%), y además con ingresos familiares mensuales entre 1 y 2 Salarios mínimos legales vigentes (SMLv) en Colombia, equivalentes menos de $\$ 510$ dólares. Lo anterior sugiere una tendencia para el género femenino con posibles cargas familiares y económicas que podrían incidir en el curso de inicio o encrudecimiento de los síntomas propios del SF.

Respecto a las características clínicas encontradas, se halló que la media de años desde que padecian la sintomatología de la enfermedad pero sin el diagnóstico fue de 6.8 años ( $D E=7.5$ ), y con el diagnóstico recibido de 3.5 años $(D E=3.8)$. Adicionalmente, las principales comorbilidades reportadas por los participantes fue la hipertensión arterial (42\%), diabetes mellitus tipo 2 (9\%), otras cardiopatías (5\%), enfermedad renal crónica (2\%), y ninguna (15\%). En la Tabla 2, se expone la tipología de los tratamientos farmacológicos que recibian al momento de realizarse la entrevista inicial.

Tabla 1.

Características sociodemográficas.

\begin{tabular}{lc}
\hline & Porcentaje (\%) \\
\hline Ocupación actual & 2 \\
Desempleado & 23 \\
Empleado & 55 \\
Ama de casa & 14 \\
Independiente & 5 \\
Pensionado por edad & 1 \\
Incapacitado & \\
Ingresos familiares promedio al mes & 25 \\
Menos de 1 smLv & 61 \\
Entre 1 y 2 smLv & 12 \\
Entre 3 y 4 smLV & 2 \\
Más de 5 sMLv & \\
Convivencia actual & 10 \\
Solo & 4 \\
Con padres & 14 \\
Con la pareja & 1 \\
Con hermanos & 32 \\
Con hijos & 39 \\
Pareja e hijos & \\
\hline
\end{tabular}

*SMLV: Salarios mínimos legales vigentes en Colombia (periodo 2017-2018).

Fuente: Elaboración propia. 
Tabla 2.

Tipología del tratamiento farmacológico en frecuencia y porcentaje.

\begin{tabular}{|c|c|c|}
\hline Tipología del Fármaco & Frecuencia (n) & Porcentaje (\%) \\
\hline Terapia biológica & 1 & 0.6 \\
\hline Fármacos antireumáticos modificadores de la enfermedad (DMARDS) & 5 & 2.8 \\
\hline Esteroides & 5 & 2.8 \\
\hline Analgésicos no esteroideos (AINES) & 47 & 26.4 \\
\hline Analgésicos opíoides & 11 & 6.2 \\
\hline Relajantes musculares & 19 & 10.7 \\
\hline Anticonvulsivantes & 17 & 9.6 \\
\hline Antidepresivos & 60 & 33.7 \\
\hline Otros & 13 & 7.3 \\
\hline
\end{tabular}

Fuente: Elaboración propia.

El reporte del tratamiento farmacológico al cual se encontraban adheridos refleja el manejo convencional en el abordaje terapéutico en la escalada del dolor, no obstante, llama la atención el elevado consumo de antidepresivos dentro del grupo (33.7 \%). Lo anterior sugirió que 6 de cada 10 participantes con SF se encontraban consumiendolos, refiriendo la afectación en la salud mental como posible consecuencia de padecer SF.

\section{Indicadores generales hallados}

En la Tabla 3, se resume los indicadores generales de depresión, ansiedad, apoyo interpersonal y dolor autopercibido hallados en los instrumentos de medición.

En los pacientes evaluados, se encontró un índice de ansiedad estado (condición emocional transitoria) muy baja, al igual que la ansiedad de

Tabla 3.

Indicadores generales.

\begin{tabular}{|c|c|c|c|c|}
\hline Instrumento & Constructo & Mínimo & Máximo & Media (DE) \\
\hline Inventario de Ansiedad-Estado (IDARE-E) & Condición emocional transitoria & 15 & 41 & $24.8(5.4)$ \\
\hline Inventario de Ansiedad-Rasgo (IDARE-R) & $\begin{array}{l}\text { Propensión ansiosa relativamente } \\
\text { estable }\end{array}$ & 9 & 48 & $26.6(7.6)$ \\
\hline Escala de depresión de Zung & Nivel de depresión & 33 & 66 & $48.7(6.8)$ \\
\hline Cuestionario de salud del paciente (PHQ-9) & $\begin{array}{l}\text { Síntomas de depresión con orien- } \\
\text { tación terapéutica }\end{array}$ & 1 & 21 & $8.2(4.2)$ \\
\hline $\begin{array}{l}\text { Escala para evaluar el apoyo interpersonal } \\
\text { ISEL-12(Total) }\end{array}$ & $\begin{array}{l}\text { Disponibilidad percibida y poten- } \\
\text { cial de los recursos sociales }\end{array}$ & 0 & 36 & $25.3(7.4)$ \\
\hline Escala visual analógica del dolor (VAS) & Escala de dolor autopercibido & 3 & 10 & $7.8(1.8)$ \\
\hline
\end{tabular}

Fuente: elaboración propia. 
rasgo (propensión ansiosa relativamente estable o continua en la persona). Indicadores de nivel de depresión moderada 48.7 ( $D E=6.8$ ), los cuales se esperaban debido a que es una de las principales características asociadas al SF. No obstante, se reportó una sintomatología depresiva de $8.2(\mathrm{DE}=4.2)$, que indicaría una orientación terapéutica basada en el juicio del clínico tratante, donde se debería profundizar en los niveles de funcionalidad y grado de afectación de manera individualizada, con el fin de determinar la posibilidad de un manejo farmacológico o psicoterapéutico.

Por otro lado, la evaluación del apoyo interpersonal, basada en la disponibilidad percibida y potencial de los recursos sociales de apoyo de las personas, se observó en niveles moderados 25.3 ( $D E=7.4$ ), lo que indicaría que el apoyo social en estos pacientes es percibido como tangible, además de sentirse moderadamente pertenecientes a una red social de apoyo, donde posiblemente pueda estar caracterizada por los miembros del mismo núcleo familiar debido a los datos sociodemográficos presentados anteriormente.

En cuanto a la autoevaluación o autopercepción del dolor en los pacientes, se observaron altos niveles de dolor reportados $7.8(D E=1.8)$. Lo anterior por ser característico del SF, pero también, posiblemente influenciados por características psicógenas como cambios con la edad, años de padecer los síntomas, aspectos educativos o socioeconómicos, acceso a los servicios de salud, dificultades familiares o de autorrealización, entre otros, que serían sin duda tema de gran interés para la investigación en salud.

\section{Asociaciones entre depresión, ansiedad, apoyo interpersonal y dolor autopercibido}

Obteniéndose los resultados de los indicadores generales, se realizó un análisis de correlación de Pearson entre las distintas medidas, sus resultados se presentan en la Tabla 4.

Se observó que la depresión, medida por la Escala de depresión de Zung, se correlacionó significativamente con la ansiedad estado y rasgo, indicando una asociación positiva, es decir, los pacientes con SF comúnmente pueden presentar ansiedad asociada a su depresión, que podría considerarse una característica más del padecimiento de la enfermedad. Adicionalmente, el apoyo interpersonal en los pacientes parece estar opuesto o verse disminuido a medida que su propensión ansiosa y niveles de depresión aumentan, lo que indicaría un posible retraimiento en sus relaciones sociales ya sea por voluntad o por exclusión de su grupo social o familiar. El dolor autopercibido, llama la atención al no asociarse con la depresión, pero si con la ansiedad estado, aunque de una manera baja y sugiriendo un posible desarrollo de desesperanza aprendida entre las personas con SF.

Tabla 4.

Correlación (Pearson) entre depresión, ansiedades, apoyo interpersonal y dolor autopercibido.

\begin{tabular}{lccccc}
\hline & $\begin{array}{c}\text { Ansiedad } \\
\text { estado }\end{array}$ & $\begin{array}{c}\text { Ansiedad } \\
\text { rasgo }\end{array}$ & Depresión & $\begin{array}{c}\text { Apoyo } \\
\text { Interpersonal }\end{array}$ & $\begin{array}{c}\text { Dolor } \\
\text { autopercibido }\end{array}$ \\
\hline Ansiedad estado & & $0.57^{* *}$ & $0.47^{* *}$ & -0.18 & $0.24^{*}$ \\
Ansiedad rasgo & $0.57^{* *}$ & & $0.62^{* *}$ & $-0.37^{* *}$ & 0.09 \\
Depresión & $0.47^{* *}$ & $0.62^{* *}$ & & $-0.22^{*}$ & 0.16 \\
Apoyo Interpersonal & -0.18 & $-0.37^{* *}$ & $-0.22^{*}$ & & 0.01 \\
Dolor autopercibido & $0.24^{*}$ & 0.09 & 0.16 & 0.01 & \\
\hline
\end{tabular}

${ }^{* *} p<0.01,{ }^{*} p<0.05$

Fuente: elaboración propia. 


\section{Discusión}

El objetivo del presente estudio fue determinar las principales características sociodemográficas y clínicas, hallar los indicadores generales de depresión, ansiedad, apoyo interpersonal, dolor autopercibido, y sus relaciones, en los pacientes diagnósticados con SF. Lo anterior con el propósito de contribuir a la caracterización y mejoramiento en la atención en salud de las posibles principales consecuencias o afectaciones de padecer este síndrome.

En cuanto a los hallazgos sociodemográficos y clínicos, se puede confirmar la evidencia acerca de que es un síndrome que presenta una mayor prevalencia en mujeres y en edades aún consideradas como productivas (Demirbag y Bulut, 2018). Se sugiere para futuras investigaciones analizar la posible asociación entre el inicio de la enfermedad o rápido encrudecimiento de los síntomas, con factores como la baja escolaridad, la ocupación previa a la aparición del síndrome, bajos ingresos económicos, historial familiar de enfermedades crónicas y del dolor, y tipos de personalidad.

Adicionalmente, el tratamiento polifarmacéutico en estos pacientes fue un común denominador, identificando los antidepresivos como la opción más frecuente pero sin mostrar altos niveles de eficacia (Loades, Sheils y Crawley, 2016). Considerándose los niveles moderados-altos de depresión entre esta población, sería pertinente para futuras investigaciones, un análisis exhaustivo de los tratamientos no farmacológicos brindados por el sistema de salud en Colombia. Lo anterior por encontrarse bibliografía acerca de los beneficios de manejo bajo psicoterapias de la cognición del dolor y estratégias de afrontamiento (Malfliet et al., 2017), ejecicios de autoayuda y cuidado médico (Clark et al., 2017), estimulación eléctrica para el dolor (Salazar, Stein, Marchese, Plentz y Pagnussat, 2017), restructuración cognitiva (McBride et al., 2017), terapia cognitivo conductual (Geraghty y Blease, 2018), del ejercicio graduado (Wilshire et al., 2018), y terapia de aceptación y compromiso (Kallesøe et al., 2016).

Los indicadores generales de depresión y ansiedad son equivalentes con los reportados en la literatura (Gracely et al., 2012; Singh y Kaul, 2018; Sitges, González-Roldán, Duschek y Montoya,
2018), pudiendo considerarse los de mayor prevalencia en este grupo de enfermedades. No obstante, esto plantea retos enormes en la actuación psicoterapéutica debido a las pérdidas laborales asociadas, retraimientos en el entorno social y de apoyo interpersonal (Arnold et al., 2008). El dolor autopercibido debería ser estudiado a mayor profundidad, ya que este factor pareciera estar íntimamente relacionado con aspectos como el estrés, la percepción acerca de un futuro desolador, y la sobrecarga en actividades cotidianas, además de su posible relación con el mismo curso de la enfermedad (Cheatham, Kolber, Mokha y Hanney, 2018; Demirbag y Bulut, 2018).

La depresión parece estar relacionada positivamente con la ansiedad tanto estado como rasgo, y negativamente con el apoyo interpersonal, sugiriendo una asociación complicada para la salud mental de los pacientes con SF como lo reportan varios autores (Gracely et al., 2012; Singh y Kaul, 2018). Aún así, es indispensable poner a prueba relaciones causaefecto para crear o fortalecer programas o protocolos de atención específicos para esta población. De esta manera, intentar garantizar una mayor efectividad en los tratamientos, ya que al conocerse el predecesor podría prevenirse una mayor cantidad de comorbilidades. Es de aclarar que en el presente estudio, la variable dolor autopercibido sólo pareció relacionarse con la ansiedad estado, por tal motivo, se sugiere continuar con estudios que indaguen sobre su relación con la depresión u otras afectaciones psicopatológicas, lo anterior por ser el dolor autopercibido una de las principales y peores características del SF (Courtois, Cools y Calsius, 2015; Gracely et al., 2012).

Es importante que los investigadores en el ára puedan replicar los estudios sobre las consecuencias de esta enfermedad en diferentes contextos y culturas, para así observar diferencias y realizar tratamientos más sensibles frente a estos factores. Adicionalmente, se requieren más investigaciones acerca del impacto y curso de la enfermedad sobre la calidad de vida en estos pacientes (Gómez, Vinaccia y Quiceno, 2017; Rojas, Zapata, Anaya y Pineda, 2005), al igual que sus posibles relaciones con factores como la depresión, la ansiedad, el estrés, el dolor autopercibido y el deterioro del apoyo social (Vélez, Palacio, Moreno y Krikorian, 2013). 
De igual forma, se sugiere que en futuras investigaciones en Colombia, se ahonde acerca de las comorbilidades médicas y los niveles de cronicidad propias del SF (Triviño, Solano y Siles, 2014), su relación e impacto con la fatiga crónica (Faro et al., 2014), los problemas de sueño asociados (Prados y Miró, 2012), y sus alteraciones en el rendimiento cognitivo (Alegre, 2008; Gelonch, Garolera, Rosselló y Pifarré, 2013; Santamarina-Pérez et al., 2011). Lo anterior por ser factores que empeoran las condiciones médicas y generan un impacto desafortunado para los pacientes y a su entorno familiar y social.

En conclusión, la salud mental, el apoyo interpersonal y el dolor autopercibido se encuentran afectados en los pacientes evaluados con SF. Por tal motivo, se requiere ajustar los protocolos de atención clínica con el propósito de conservar y potenciar la salud integral en los afectados, debiendo ser interdisciplinar y propendiendo por el fortalecimiento de las redes de apoyo en el manejo del dolor, el apoyo interpersonal y las actividades de la vida cotidiana y laboral.

Dentro de las principales limitaciones del presente estudio, se puede indicar que a pesar de existir un tamaño muestral idóneo, se requiere de estudios epidemiológicos sobre la prevalencia real de personas con sf en la ciudad de Neiva y del resto del país, con el fin de utilizar técnicas de muestreo estadisticamente más solidas. Adicionalmente, factores como las instalaciones donde se realizó el proceso de aplicación del protocolo de investigación y el tiempo de ejecución, pudieron haber incidido en las respuestas de las personas evaluadas debido a la misma sitomatología de dolor y fatiga que puede producir las posturas corporales durante tiempo prolongado y su posible relación con el nivel de concentración.

En consecuencia, este estudio surge de la necesidad de fomentar y fortalecer una cultura crítica sobre la forma de hacer la atención clínica en el país y en Latinoamérica, en donde, no sólo se exponen importantes datos relacionados con las condiciones de salud en pacientes con SF y que pueden llegar a fortalecer el diagnóstico y tratamiento integral de la enfermedad, sino que pretende aportar un carácter científico y preciso que influya directamente a la sociedad a través del robustecimiento de las ciencias de la salud.

\section{Agradecimientos}

Los autores agradecen a cada una de las personas que conformaron la muestra y a sus familias, las cuales dieron su aporte desinteresadamente con el único propósito de contribuir a la ciencia, la salud y bienestar de muchas otras personas.

\section{Referencias}

Ahlers, S., Van der Veen, A., Van Dijk, M., Tibboel, D. y Knibbe, C. (2010). The use of the Behavioral Pain Scale to assess pain in conscious sedated patients. Anesthesia y analgesia, 110(1), 127-133. https://doi.org/10.1213/ ANE.0b013e3181c3119e

Alegre, J. (2008). La Fibromialgia es una entidad primaria del dolor: ¿Hay deterioro cognitivo en la Fibromialgia?. Revista de la Sociedad Española del Dolor, 15(6), 355-357.

Amaro, T., Martín, J., Anton, P. y Granados, J. (2006). Fibromialgia: estudio de la calidad de vida antes y después del tratamiento psicológico. Cuadernos de medicina psicosomática y psiquiatría de enlace, 79, 47-52.

Arnold, L., Crofford, L., Mease, P., Burgess, S., Palmer, S., Abetz, L. y Martin, S. (2008). Patient perspectives on the impact of fibromyalgia. Patient Education and Counseling, 73(1), 114120. https://doi.org/10.1016/j.pec.2008.06.005

Arnold, L., Hudson, J., Hess, E., Ware, A., Fritz, D., Auchenbach, M., ... Keck, P. (2004). Family study of fibromyalgia. Arthritis \& Rheumatism, 50(3), 944-952. https://doi.org/10.1002/art.20042

Briones-Vozmediano, E., Ronda-Pérez, E. y VivesCases, C. (2015). Percepciones de pacientes con fibromialgia sobre el impacto de la enfermedad en el ámbito laboral. Atención primaria, 47(4), 205-212. https://doi.org/10.1016/j. aprim.2014.06.002

Cabo-Meseguer, A., Cerdá-Olmedo, G. y TilloMatta, J. (2017). Fibromialgia: prevalencia, perfiles epidemiológicos y costes económicos. 
Medicina clínica, 149(10), 441-448. https://doi. org/10.1016/j.medcli.2017.06.008

Campo, A., Díaz, L. y Rueda, G. (2006). Validez de la escala breve de Zung para tamizaje del episodio depresivo mayor en la población general de Bucaramanga, Colombia. Biomédica, 26(3), 415-23.

Cardona-Arias, J., León-Mira, V. y Cardona-Tapias, A. (2013). Calidad de vida relacionada con la salud en adultos con fibromialgia. Revista colombiana de Reumatología, 20(1), 19-29. https://doi.org/10.1016/S0121-8123(13)70009-4

Cardona-Arias, J., León-Mira, V. y Cardona-Tapias, A. (2014). Estado de salud y calidad de vida en pacientes con fibromialgia, Medellín. Revista Colombiana de Reumatología, 21(1), 10-20. https://doi.org/10.1016/S0121-8123(14)70142-2

Casado, V. (2015). La fibromialgia. del malestar al bienestar: estilos de vida saludables. Anales de la Real Academia de Medicina y Cirugía de Valladolid, 52, 47-56.

Cheatham, S., Kolber, M., Mokha, M. y Hanney, W. (2018). Validez concurrente de las escalas de dolor en individuos con dolor miofascial y fibromialgia. Journal of Manual y Manipulative Therapy, 26(1), 25-35. https://doi.org/10.1080 /10669817.2017.1349592

Clark, L., Pesola, F., Thomas, J., Vergara-Williamson, M., Beynon, M. y White, P. (2017). Guided graded exercise self-help plus specialist medical care versus specialist medical care alone for chronic fatigue syndrome (GETSET): a pragmatic randomised controlled trial. The Lancet, 390(10092), 363-373. https://doi.org/10.1016/ S0140-6736(16)32589-2

Courtois, I., Cools, F. y Calsius, J. (2015). Effectiveness of body awareness interventions in fibromyalgia and chronic fatigue syndrome: A Systematic Review and Meta-Analysis. Journal of Bodywork and Movement Therapies, 19(1), 35-56. https://doi.org/10.1016/j. jbmt.2014.04.003
De Loach, L., Higgins, M., Caplan, A. y Stiff, J. (1998). The Visual Analog Scale in the immediate postoperative period: intrasubject variability and correlation with a numeric scale. Anesthesia y Analgesia, 86(1), 102-106. https:// doi.org/10.1213 / 00000539-199801000-00020

Demirbag, B. y Bulut, A. (2018). Demographic characteristics, clinical findings and functional status in patients with fibromyalgia syndrome. Journal of the Pakistan Medical Association, 68(7), 1043-1047.

Faro, M., Sáez-Francàs, N., Castro-Marrero, J., Aliste, L., Collado, A. y Alegre, J. (2014). Impacto de la fibromialgia en el síndrome de fatiga crónica. Medicina Clínica, 142(12), 519-525. https://doi. org/10.1016/j.medcli.2013.06.030

Gelonch, O., Garolera, M., Rosselló, L. y Pifarré, J. (2013). Disfunción cognitiva en la fibromialgia. Revista de Neurología, 56(11), 573-588. https:// doi.org/10459.1/63103

Geraghty, K. y Blease, C. (2018). Cognitive behavioural therapy in the treatment of chronic fatigue syndrome: A narrative review on efficacy and informed consent. Journal of Health Psychology, 23(1), 127-138. https://doi. org/10.1177/1359105316667798

Gómez, C., Vinaccia, S. y Quiceno, J. (2017). Enfermedades reumáticas en Colombia: quince años de investigaciones psicológicas. Universitas Médica, 58(2). https://doi. org/10.11144/Javeriana.umed58-2.reum

Gracely, R., Ceko, M. y Bushnell, M. (2012). Fibromyalgia and depression. Pain research and treatment, 2012, ID: 486590. https://doi. org/10.1155/2012/486590

Haworth Editorial Submission. (1993). Consensus document on fibromyalgia: the Copenhagen Declaration. Journal of musculoskeletal pain, 1(3-4), 295-312. https://doi.org/10.1300/ J094v01n03_33

Kallesøe, K., Schröder, A., Wicksell, R., Fink, P., Ørnbøl, E. y Rask, C. (2016). Comparing 
group-based acceptance and commitment therapy (ACT) with enhanced usual care for adolescents with functional somatic syndromes: a study protocol for a randomised trial. BMJ open, 6(9), e012743. https://doi.org/10.1136/ bmjopen-2016- 012743

Kato, K., Sullivan, P., Evengård, B. y Pedersen, N. (2006). Importance of genetic influences on chronic widespread pain. Arthritis \& Rheumatism: Official Journal of the American College of Rheumatology, 54(5), 1682-1686. https://doi.org/10.1002/art.21798

Lautenbacher, S., Rollman, G. y McCain, G. (1994). Multi-method assessment of experimental and clinical pain in patients with fibromyalgia. Pain, 59(1), 45-53. https://doi. org/10.1016/0304-3959(94)90046-9

Loades, M., Sheils, E. y Crawley, E. (2016). Treatment for pediatric chronic fatigue syndrome or myalgia encephalomyelitis (CFS/ME) and comorbid depression: a systematic review. BMJ Open, 6(10), e012271. https://doi.org/10.1136/ bmjopen-2016-012271.

López, M. y Mingote, J. (2008). Fibromyalgia. Clínica y Salud, 19(3), 343-358.

Maletic, V. y Raison, C. (2009). Neurobiology of depression, fibromyalgia and neuropathic pain. Frontiers in Bioscience, 14, 5291-5338. https:// doi.org/10.2741/3598

Malfliet, A., Coppieters, I., Van Wilgen, P., Kregel, J., De Pauw, R., Dolphens, M. y Ickmans, K. (2017). Brain changes associated with cognitive and emotional factors in chronic pain: a systematic review. European Journal of Pain, 21(5), 769-786. https://doi.org/10.1002/ejp.1003

McBride, R., Horsfield, S., Sandler, C., Cassar, J., Casson, S., Cvejic, E., ... Lloyd, A. (2017). Cognitive remediation training improves performance in patients with chronic fatigue syndrome. Psychiatry Research, 257, 400-405. https://doi.org/10.1016/j. psychres.2017.08.035
Moreno, J., Arango-Lasprilla, J. y Rogers, H. (2010). Necesidades familiares y su relación con las características psicosociales que presentan los cuidadores de personas con demencia. Psicología desde el Caribe, 26, 1-35.

Pérez, J. (2005). Manejo del estrés en estudiantes universitarios para reducir la ansiedad en exámenes (Tesis de maestría, Universidad de las Américas). Recuperado de: http://catarina.udlap.mx/u_dl_a/tales/documentos/moor/ perez_a_jl/resumen.html

Prados, G. y Miró, E. (2012). Fibromialgia y sueño: una revisión. Revista de Neurología, 54(4), 227240. https://doi.org/10.33588/rn.5404.2011556

Revuelta-Evrard, E., Segura-Escobar, E. y PaulinoTevar, J. (2010). Depresión, ansiedad y fibromialgia. Revista de la Sociedad Española del Dolor, 17(7), 326-332. https://doi.org/10.1016/j. resed.2010.07.002

Rojas, A., Zapata, A. L., Anaya, J. y Pineda, R. (2005). Predictores de calidad de vida en pacientes con fibromialgia. Revista Colombiana de Reumatología, 12(4), 295-300.

Salazar, A., Stein, C., Marchese, R., Plentz, R. y Pagnussat, A. (2017). Electric Stimulation for pain relief in patients with Fibromyalgia: A Systematic Review and Meta-analysis of randomized controlled trials. Pain Physician, 20(2), 15-25.

Santamarina-Perez, P., Freniche, V., Eiroa-Orosa, F., Llobet, G., Saez, N., Alegre, J. y Jacas, C. (2011). El rol de la depresión en el déficit cognitivo del paciente con síndrome de fatiga crónica. Medicina Clínica, 136(6), 239-243. https:// doi.org/10.1016/j.medcli.2010.07.022

Silber, E. y Tippett, J. (1965). Self-esteem: Clinical assessment and measurement validation. Psychological Reports, 16(3_suppl), 1017-1071. https://doi.org/10.2466/pr0.1965.16.3c.1017

Singh, G. y Kaul, S. (2018). Anxiety and depression are common in fibromyalgia patients and 
correlate with symptom severity score. Indian Journal of Rheumatology, 13(3), 168-172. https://doi.org/10.4103 / injr.injr_52_18

Souza, V., Zortea, M., Alves, R., Cilene, C., Naziazeno, S., Saldanha, J., ... Caumo, W. (2018). Cognitive effects of transcranial direct current stimulation combined with working memory training in fibromyalgia: a randomized clinical trial. Scientific reports, 8(1), 1-11. https://doi.org/10.1038/s41598-018-30127-z

Spielberger, C. y Guerrero-Díaz, R. (1975). IDARE: Inventario de Ansiedad: Rasgo-Estado. Manual e instructivo. Ciudad de México: Manual Moderno.

Sitges, C., González-Roldán, A., Duschek, S. y Montoya, P. (2018). Emotional influences on cognitive processing in fibromyalgia patients with different depression levels. The Clinical Journal of Pain, 34(12), 1106-1113. https://doi. org/10.1097/AJP.0000000000000637.

Triviño, Á., Solano, M. y Siles, J. (2014). La cronicidad de la fibromialgia: una revisión de la literatura. Enfermería Global, 13(35), 273-292. https://doi.org/10.6018/eglobal.13.3.182621
Vélez, M., Palacio, C., Moreno, A. y Krikorian, A. (2013). Psychological and family-related facts of suffering in patients with chronic diseases. Techniques in Regional Anesthesia and Pain Management, 17(1), 7-10. https://doi. org/10.1053/j.trap.2013.09.002

Vicente-Herrero, M., Terradillos-García, M., Capdevila-García, L., Ramírez-Íñiguez de la Torre, M. y López-González, A. (2011). Fibromialgia y trabajo. Valoración en la legislación española. Revista Médica del Instituto Mexicano del Seguro Social, 49(5), 511-516.

Wilshire, C., Kindlon, T., Courtney, R., Matthees, A., Tuller, D., Geraghty, K. y Levin, B. (2018). Rethinking the treatment of chronic fatigue syndrome-a reanalysis and evaluation of findings from a recent major trial of graded exercise and CBT. BMC psychology, 6(1), 6. https:// doi.org/10.1186/s40359-018-0218-3

Wulsin, L., Somoza, E. y Heck, J. (2002). The feasibility of using the Spanish PHQ-9 to screen for depression in primary care in Honduras. Primary Care Companion to the Journal of Clinical Psychiatry, 4(5), 191-195. https://doi.org/10.4088 / pcc.v04n0504 